$16^{\text {th }}$ International Congress of Metrology, 11001 (2013)

DOI: $10.1051 /$ metrology/201311001

(C) Owned by the authors, published by EDP Sciences, 2013

\title{
AIM QUTE: Automated Impedance Metrology extending the Quantum Toolbox for Electricity
}

\author{
L. Palafox ${ }^{1, \mathrm{a}}$, F. Raso ${ }^{2}$, J. Kučera ${ }^{3}$, F. Overney ${ }^{4}$, L Callegaro ${ }^{5}$, P. Gournay ${ }^{6}$, A. Ziołek ${ }^{7}$, J. Nissilä ${ }^{8}$, G. Eklund ${ }^{9}$, T. Lippert ${ }^{10}$, \\ Y. Gülmez ${ }^{11}$, P. Fleischmann ${ }^{12}$, M. Kampik ${ }^{13}$, R. Rybski ${ }^{14}$ \\ ${ }^{1}$ Physikalisch-Technische Bundesanstalt, Bundesallee 100, D-38116 Braunschweig, Germany \\ ${ }^{2}$ Centro Español de Metrología, C/del Alfar 2, E-28760 Tres Cantos (Madrid), Spain \\ ${ }^{3}$ Cesky Metrologicky Institut, Okružní 31, CZ-638 00 Brno, Czech republic \\ ${ }^{4}$ METAS, Swiss Federal Office of Metrology, Lindenwe 50, CH-3003 Bern-Wabern, Switzerland \\ ${ }^{5}$ Istituto Nazionale di Ricerca Metrologica, Strada delle Cacce 91, IT-10135 Torino, Italy \\ ${ }^{6}$ Laboratoire National de Métrologie et d'Essais (LNE), 29 avenue Roger Hennequin, F-78197 Trappes Cedex, France \\ ${ }^{7}$ Główny Urząd Miar, ul. Elektoralna 2, 00-139 Warszawa ,Poland \\ ${ }^{8}$ MIKES, Otakaari 7B, FIN-02150 Espoo, Finland \\ ${ }^{9}$ SP Technical Research Institute of Sweden, Box 857, SE-501 15 Borås, Sweden \\ ${ }^{10}$ Trescal A/S, Mads Clausens Vej 12, DK-8600 Silkeborg, Denmark \\ ${ }^{11}$ Turkiye Bilimsel ve Teknolojik Arastirma Kurumu, Ataturk Bulvari 221, TR-06100 Ankara, Turkey \\ ${ }^{12}$ esz AG calibration \& metrology, Max-Planck-Strasse 16, D-82223 Eichenau, Germany \\ ${ }^{13}$ Politechnika Slaska, ul. Akademicka 10, 44-100 Gliwice, Poland \\ ${ }^{14}$ Uniwersytet Zielonogorski, ul. Podgórna 50, 65-246 Zielona Góra, Poland
}

\begin{abstract}
The "Automated impedance metrology extending the quantum toolbox for electricity" project (AIM QuTE) will extend Josephson impedance bridges capabilities to the whole complex plane with the best level of uncertainties $(0.05 \mathrm{ppm})$. In parallel, fully digital bridges will be developed to reduce the operator workload imposed on national metrology institutes for the realisation of the impedances scales and for calibrations. The target level of uncertainty for these fully digital bridges is parts in $10^{-7}$. Furthermore, sub-pF standards will be developed to establish traceability of very small capacitances. An electronic impedance simulator, which will cover a very large region of the complex impedance plane, will also be realised, it will significantly reduce the number of standards required to link traceability of top level calibration laboratories to national metrology institutes.
\end{abstract}

\section{Introduction}

Impedance is central in electrical metrology and also in many other disciplines that use impedance changes in sensors for their measurements. Dissemination is presently based on manually operated coaxial bridges that can only be used over a limited range of frequencies. We will present a joint research project (JRP) in the European metrology research programme or EMRP framework that will make the transition from measurements performed at predefined values and relative phase angles for selected frequencies to any ratio over the complete audio frequency range $(20 \mathrm{~Hz}$ to $20 \mathrm{kHz})$. This transition will be achieved with two different types of impedance bridges: Josephson bridges and digital bridges. In addition to developing these novel types of bridges, we will also build standards required to extend the impedance scales to non-decadic values for "pure" impedances $(R, L$ and $C)$, to small capacitances as

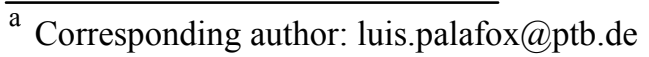

encountered in nanotechnology, and to establish the capability of the novel bridges to measure at intermediate phase angles without a significant loss in accuracy. The project is called "Automated Impedance Metrology extending the Quantum Toolbox for Electricity", or AIM QuTE in short.

\section{Project background}

At present, impedance standards are maintained by numerous artefacts to cover a wide range of values. The chain of calibrations needed for the generation of an impedance scale and the link between different impedance types - resistance, capacitance and inductance - is achieved, at the NMI (National Metrology Institute) level, with transformer based coaxial bridges. All these measurements are performed at a single frequency and the bridge needs to be recalibrated for each decade of 
capacitance. In their classical implementations, coaxial bridges employ a number of fixed and variable transformers and inductive voltage dividers. Their complexity requires a highly skilled operator to operate and balance these coaxial bridges. Attempts to automate these bridges invariably result in larger uncertainties due to the inherent limitations introduced by controlling these variable devices remotely.

This JRP will advance beyond this state of the art in multiple areas. In the first place, it will extend Josephson impedance bridges e.g. to measurements:

- along the axes of the complex plane of impedance $(R: R, C: C$ and $L: L)$ - arbitrary ratios of like impedances -,

- between the axes - quadrature bridges,

- and at arbitrary phase angles.

In second place, fully digital bridges will be developed to reduce the operator workload imposed on NMIs for the realisation of the impedance scales and for calibrations. The target level of uncertainty for these fully digital bridges is parts in $10^{7}$.

The infrastructure for impedance will furthermore be improved with the active and passive impedance standards manufactured. Sub-pF standards are required to establish traceability for this end of the impedance scales, where there are none at the moment. In addition, an electronic impedance simulator will cover a very large region of the complex impedance plane and significantly reduce the number of standards that are required to link the traceability of top level calibration laboratories to NMIs.

\section{The need for the project}

Impedance is used directly in some applications such as the manufacture of passive electronic components and indirectly to measure a huge number of other parameters: resistance based temperature metrology, electrochemical impedance spectroscopy for the analysis of battery electrolytes, calibration of dosimeters for ionizing radiation, sound and vibration instrumentation, and commercial sensors such as touchscreens or fuel gauges. Both "end users" of impedance measurements and

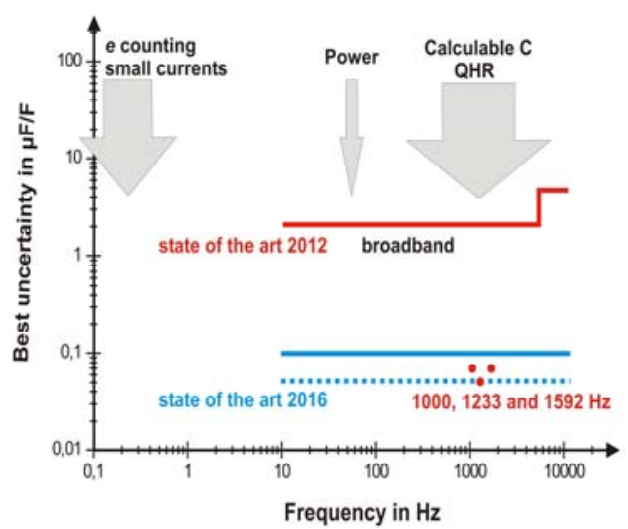

Figure 1. Uncertainty as a function of frequency for the calibration of a $100 \mathrm{pF}$ capacitance standard. instrument manufacturers have identified areas in need of improvement.

Up to now, the lowest uncertainties for impedance calibrations have been restricted to previously defined ratios and phase angles. An example can be seen in figure 1 for the state of the art uncertainty as a function of frequency for a capacitance standard of $100 \mathrm{pF}$. Calibrations are performed as a comparison with a known impedance. The ratio is normally defined by a purposebuilt transformer. State of the art transformers enable the two impedances to be compared with uncertainties of a few parts in $10^{8}$ or better over a limited frequency range (at best $500 \mathrm{~Hz}$ to $5 \mathrm{kHz}$ ). A different transformer is required for a different impedance ratio. Both the construction of the ratio transformers and balancing the bridge for the calibrations require highly skilled operators. The impedance values that can be measured with high accuracy (parts in $10^{8}$ ) are the decadic values and the quantum Hall resistance (QHR) values. Currently the uncertainty is even larger for impedances that are exactly on the axes or very close to them. The situation is depicted in figure 2 .

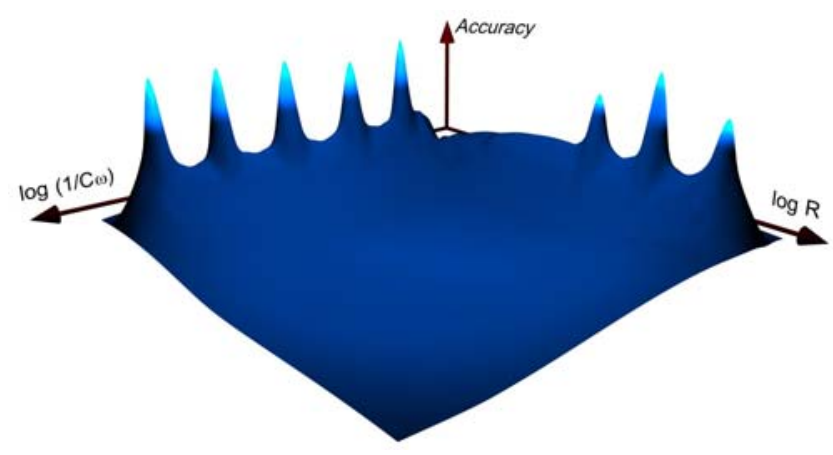

Figure 2. Artist's impression of the best possible accuracy as a function of impedance value at $1 \mathrm{kHz}$ in the subplane between the capacitance and resistance axes before the JRP.

The novel impedance bridges that will be developed in the JRP will allow measuring impedances over the complete complex plane with the same uncertainties, as illustrated in figure 3 .

These novel capabilities require the construction of a number of passive standards in order to validate these unprecedented low uncertainties for arbitrary impedance ratios along the axes and for intermediate phase angles. In addition, a novel programmable impedance simulator will be built in the JRP. The impedance simulator is an electronic instrument that can be programmed to behave,

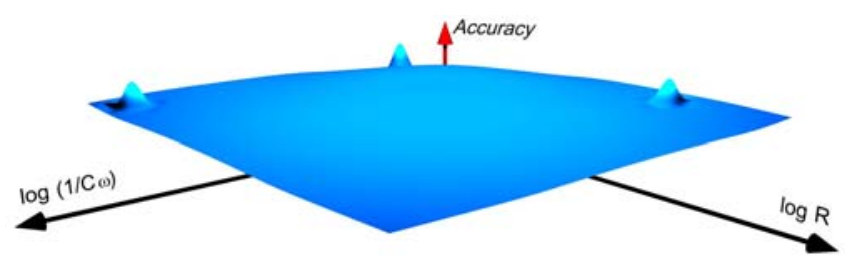

Figure 2. Artist's impression of the best possible accuracy as a function of impedance value at $1 \mathrm{kHz}$ in the subplane between the capacitance and resistance axes at the end of the JRP. 
in the frequency range between $50 \mathrm{~Hz}$ and $20 \mathrm{kHz}$, as a four terminal pair impedance with any magnitude between $10 \Omega$ and $1 \mathrm{M} \Omega$ and an arbitrary phase angle. The impedance simulator has the potential of substituting the large number of standards that are required at the moment in order to transfer the impedance scales to calibration laboratories.

\section{Scientific objectives}

The focus of this JRP is to provide the tools and methods needed to establish the impedance scale. The aim is to meet user requirements for reliable impedance measurements in the whole complex plane, in the frequency range between $10 \mathrm{~Hz}$ and $20 \mathrm{kHz}$.

The JRP addresses the following scientific and technical objectives:

- Realisation of Josephson based impedance bridges for arbitrary ratios of like impedances $(R: R, C: C$ and possibly $L: L$ ), and $1: 1$ ratios for quadrature measurements. By the end of the project, four partner NMIs and a collaborator will operate Josephson impedance bridges.

- Development and proof-of-concept tests of automated impedance ratio bridges at an uncertainty level of $10^{-7}$ covering the frequency range from $10 \mathrm{~Hz}$ to $20 \mathrm{kHz}$.

- Extend the impedance scales to intermediate values along the axes $(R, L$ and $C)$, to intermediate phase angles and towards values demanded by nanotechnology (capacitances below fF), and the corresponding standards developed. The European metrology infrastructure will be improved as a result.

\section{Expected results}

The direct impact of the project will be mainly regarding traceability and NMI capabilities. New routes will be established where there are none at the moment (for impedance values commonplace in nanotechnology). The extension of NMI calibration capabilities to intermediate phase angles will also allow confirmation of industrial calibration of standards. Automated impedance bridges for unprecedented accuracies will also reduce the costs of calibrations. At least nine NMIs will operate automated bridges by the end of the project; five of them based on Josephson arrays. Four partners will develop and test fully digital bridges that also operate over a comparable frequency range and without the need for a highly skilled operator. Additional potential for reductions in the complexity and costs associated with today's maintenance of the impedance scales will be realized through the programmable impedance simulator developed in this JRP.

Furthermore, the new, broadband impedance bridges from this JRP will enable a more precise understanding of the reported difference in value of capacitance standards between $\mathrm{DC}$ and $\mathrm{AC}$, with a direct impact in the "mise en pratique" for capacitance and current.

The partners in the JRP will be in close contact with stakeholders interested in the application of the results from the project. The consortium has identified twenty four interested stakeholders that are distributed in Europe, North America and Asia; fourteen companies, four research institutions and six NMIs.

Indirect impact towards manufacturers of passive electronic components, instrumentation manufacturers for impedance measuring equipment and their users will also benefit from the automated operation of the bridges developed in the JRP.

The project will promote the dissemination of quantum based standards, following in the footsteps of the iMERA-Plus JRP "JoSy".

\section{Acknowledgements}

The AIM QuTE project is part of the EMRP. The EMRP is jointly funded by the EMRP participating countries and the European Union. 